\title{
Compatibility of metal additive manufactured tungsten collimator for SPECT/MRI integration
}

\author{
Amine M Samudi ${ }^{1 *}$, Karen Van Audenhaege ${ }^{2}$, Gunter Vermeeren ${ }^{1}$, Luc Martens ${ }^{1}$, Roel Van Holen², Wout Joseph ${ }^{1}$ \\ From PSMR 2015: 4th Conference on PET/MR and SPECT/MR \\ La Biodola, Isola d'Elba, Italy. 17-21 May 2015
}

${ }^{1}$ INTEC, Ghent University/iMinds, Ghent, Belgium
We optimized the MR-compatibility of a novel tungsten collimator, produced with metal additive manufacturing that is part of a microSPECT insert for a preclinical SPECT/MRI scanner. We characterized the current density due to the gradient field and adapted the collimators by smart design to reduce the induced eddy currents. The z-gradient coil and the collimator were modeled with SEMCAD. The gradient strength was $510 \mathrm{mT} / \mathrm{m}$, the gradient efficiency was about $3.4 \mathrm{mT} / \mathrm{m} / \mathrm{A}$. The setup was simulated with a working frequency of $10 \mathrm{kHz}$. The system consists of 7 identical collimators and digital silicon photomultipliers assembled in a ring. We evaluated the global reduction in current density J (reduction) based on the sum of all current densities in the collimator. We applied the following optimizations on the collimator: 1 . We reduced the excessive material in the flanges. 2 . We applied horizontal slits of $2 \mathrm{~mm}$ in the collimator surface. 3 . We reduced material in the core; the photons are attenuated before they reach the core. The collimator will need a supporting structure. 4 . The supporting structure can be avoided by using two vertical slits in the middle of the collimator. 5. We used a Z-shaped slit instead of the vertical slit. Results of simulations show that smaller flanges reduce the current density with $23 \%$. The horizontal slits reduce the eddy currents with $6 \%$. Using less material in the core or applying vertical slits results in the same reduction of current density. However, the vertical slits are cheaper because a hollow collimator requires supporting structures during production. Both can be combined if $\mathrm{z}$-shaped slits are used to prevent attenuation problems. The reduction is then $27 \%$. Finally, when all previous adaptations are combined, the reduction in eddy currents is about $56.3 \%$.

Authors' details

${ }^{1}$ INTEC, Ghent University/iMinds, Ghent, Belgium . ${ }^{2}$ ELIS, Ghent University/iMinds, Gent, Belgium

Published: 18 May 2015

doi:10.1186/2197-7364-2-S1-A52

Cite this article as: Samudi et al.: Compatibility of metal additive manufactured tungsten collimator for SPECT/MRI integration. EJNMMI Physics 2015 2(Suppl 1):A52.

\section{SpringerOpen ${ }^{\circ}$}

(C) 2015 Samudi et al; licensee Springer. This is an Open Access article distributed under the terms of the Creative Commons Attribution License (http://creativecommons.org/licenses/by/4.0), which permits unrestricted use, distribution, and reproduction in any medium, provided the original work is properly cited. 